\title{
Audiometric screening of a population with intellectual disability
}

Eva Andersson, Stig Arlinger, Lennart Magnusson and Elisabeth Hamrin

\section{Linköping University Post Print}

\section{Tweet}

N.B.: When citing this work, cite the original article.

Original Publication:

Eva Andersson, Stig Arlinger, Lennart Magnusson and Elisabeth Hamrin, Audiometric screening of a population with intellectual disability, 2013, International Journal of Audiology, (52), 1, 50-56.

http://dx.doi.org/10.3109/14992027.2012.700773

Copyright: Informa Healthcare http://informahealthcare.com/

Postprint available at: Linköping University Electronic Press http://urn.kb.se/resolve?urn=urn:nbn:se:liu:diva-87267 


\section{AUDIOMETRIC SCREENING OF A POPULATION WITH INTELLECTUAL DISABILITY}

Eva Andersson ${ }^{1}$

Stig Arlinger ${ }^{1}$

Lennart Magnusson ${ }^{2}$

Elisabeth Hamrin ${ }^{3}$

${ }^{1}$ Department of Clinical and Experimental Medicine, Faculty of Health Sciences, Linköping University, Linköping, Sweden

${ }^{2}$ Department of Audiology, Sahlgrenska University Hospital, Gothenburg, Sweden

${ }^{3}$ Department of Medical and Health Sciences, Faculty of Health Sciences, Linköping University, Linköping, Sweden

\section{KEY WORDS}

intellectual disability, screening audiometry, psycho-acoustic method

\section{ABBREVIATIONS}

ABR - auditory brainstem response

BOA - behavioural observation audiometry

CPA - conditioned play audiometry

DS - Down syndrome

ENT - ear nose and throat

$\mathrm{HL}$ - hearing level

HTL - hearing threshold level

ID - intellectual disability 
$\mathrm{OAE}$ - oto-acoustic emission

OPTA - ordinary pure-tone audiometry

PTA - pure-tone audiometry

TEOAE - transient evoked oto-acoustic emission

VRA - visual reinforcement audiometry

\section{CORRESPONDING AUTHOR}

Eva Andersson

Gärdesvägen 29B

S-436 51 Hovås

Sweden

eva.bm.andersson@telia.com 
ABSTRACT

Objective: Evaluation of pure-tone audiometry (PTA) in hearing screening of a population with mild to profound intellectual disability (ID).

Design: PTA was performed at six frequencies at the screening level $20 \mathrm{~dB}$ HL. Referral criteria were threshold levels $\geq 25 \mathrm{~dB}$ HL at two or more frequencies for one ear or both. Study sample: 1478 participants in ages 7-91 were included.

Results: 1470 (99.5\%) people cooperated in screening of which $1325(90 \%)$ could be tested on both ears at all six frequencies. A majority, 987 (66.8\%), performed ordinary PTA, 234 (15.8\%) conditioned play audiometry, and 249 (16.9\%) behavioural observation audiometry. $669(45 \%)$ passed and $809(55 \%)$ failed according to referral criteria. Of those failing, 441 $(54.5 \%)$ accepted referral to clinical evaluation.

Conclusions: PTA with slight modifications is applicable for screening of a population with mild to profound intellectual disability. The most challenging and time-consuming activity is to introduce the test procedure in a way that reduces anxiety and establishes trust. 


\section{AUDIOMETRIC SCREENING OF A POPULATION WITH INTELLECTUAL DISABILITY}

An intellectual disability (ID) is frequently associated with communication difficulties, and an ID in combination with a temporary or permanent hearing impairment (HI) involves considerable communicative impediments for the affected individual. These problems will be even more accentuated if additional disabilities occur. However, if the hearing problems are minimized, the capability for communication expands, reducing the negative psychological and social consequences and improving the quality of life (Fulton \& Lloyd, 1975; van Schrojenstein Lantman-de Valk et al., 1994; Evenhuis et al., 2001). Therefore, access to hearing investigations including standardized, universal, recurrent hearing screening must be offered people with intellectual disability.

\section{Screening methods}

General criteria for an effective screening method are to simply, rapidly, non-invasively and cheaply separate the participants into two groups - pass and fail - according to a stipulated screening level (Wilson \& Jungner, 1968; Davis et al., 1997). The most common hearing screening methods fulfil these criteria. Recording of otoacoustic emissions (OAE) and automated auditory brainstem responses (ABR) are successfully used for neonatal hearing screening (White et al., 1994), while pure tone audiometry (PTA) is usually preferred for hearing screening of older children and adults, e.g. for hearing tests on school-children and on noise-exposed populations (American Speech-Language-Hearing Association, 1997;

Rabinowitz et al., 2011).

Screening by PTA provides frequency specific results as the best basis for assessing the need for further interventions, while a limitation of the neonatal screening methods is the lack of 
frequency specific information regarding estimated hearing thresholds, since broad-band clicks is the normal type of stimulation.

\section{Hearing investigations - Previous research}

A literature survey of hearing investigations for screening and/or diagnostic purposes among older children and adults with ID indicates a methodological diversity and an infrequent use of pure-tone audiometry (PTA) - gold standard for audiometry in general.

Lavis et al (1997) published a historical review of Australian, UK and US studies with varying approaches. The 16 studies, which were published from 1971 to 1995, included from 53 to 98034 participants from school age to adults, with different kinds and degrees of ID. Nine of the investigations were performed by "audiological assessment", including a variety of methods from pure-tone audiometry to distraction/localising testing, "brief audiological screening by hospital nurses" or "simple clinical methods". Seven of the investigations were performed by questionnaires given to caregivers. The prevalence of hearing loss ranged from 7 to $47 \%$, except for one study of people with Down syndrome (DS), which showed a prevalence of as much as $73 \%$.

Lavis et al (1997) compared prevalence results from systematic hearing assessment and a staff opinion survey in 324 institutionalized adults with ID. The assessment included PTA and/or informal tests, but they did not report how many were able to co-operate in PTA. The prevalence of hearing loss by hearing assessment was $38.9 \%$ but only $9.3 \%$ in the survey, and the authors stressed the need for audiometric assessment by experienced testers. 
In the majority of recent studies, emphasis seems to be shifting to physiological screening methods like automated recording of OAEs, often supplemented with tympanometry for those failing and in some studies complemented by PTA screening for those still failing. Driscoll et al (2002) intended to test 489 intellectually disabled children in special schools using transient evoked otoacoustic emission (TEOAE) recording and tympanometry. TEOAE recording was accepted by $80 \%$ of which $40 \%$ failed, while $74 \%$ accepted tympanometry of which $25 \%$ failed. From this comprehensive study, Driscoll et al (2003) selected and focused on 27 children with DS. The test protocol was the same - TEOAE recording and tympanometry - but the percent failing was $85.2 \%$. PTA was not performed in any of the two Driscoll studies and it was doubted that "gold standard audiological data" from special school populations could be achieved.

Meuwese-Jongejeugd et al (2006) presented a population-based prevalence study among people with ID. Out of a random sample of 2706 people, 1598 subjects (59\%) were tested. "Reliable audiometric data" were obtained from 1215 (76\%). These results were based on PTA in 831 cases (52\%) and on OAE recording in 384 cases (24\%). Passing in OAE recording bi- or unilaterally was by definition considered as no hearing impairment.

Neumann et al (2006), Hild et al (2008) and Kumar Sinha et al (2008) reported hearing screening in the Healthy Athletes Program. Volunteers performed the screenings in four steps following international guidelines. Initial otoscopy was followed by TEOAE-recording and, if failing, tympanometry and PTA at 2 and $4 \mathrm{kHz}$ with screening level $25 \mathrm{~dB} \mathrm{HL}$. If still failing, screening at all frequencies was offered, and, if indicated, consultation by an ear-nose- throat (ENT) specialist was recommended. The studies included 524 to 855 people, and of them 23.5 
$-42 \%$ failed. As pointed out by Neumann et al, it is important to consider that OAErecordings only show the peripheral function of the auditory system.

\section{Classification of ID}

A population with ID is not homogeneous. There are individual differences in aetiology, degree of ID, degree of communication difficulties, additional disabilities as well as differences in life history and lived experiences. These factors either restrict or optimize the capacity of the individual, and the developmental profile may be uneven, e.g. imperfect intellectual capacity in combination with adequate social behaviour. However, developmentally oriented researchers emphasize that people with ID in general take the same developmental steps as people of average intelligence but reach their optimal capacity on a lower level (Greenspan \& Granfield, 1992; Hodapp et al, 1998; Granlund, 1993).

Despite the complex picture from the individual perspective, the conventional description of intellectual ability is the intelligence quotient (IQ). The IQ can be expressed by referring to a mental age, estimated by standardized intelligence tests. WHO (2010) proposed an IQ-based classification as follows: mild ID refers to a mental age of 9-12 years, moderate ID to 6-9 years, severe ID to 3-6 years, and profound ID to a mental age of $<3$ years. In Sweden, the prevalence of ID amounts to $0.4 \%$ or 40.000 people, i.e. the number of intellectually disabled registered to receive special support and service. Approximately $24 \%$ are mildly, $34 \%$ moderately and $41 \%$ severely or profoundly disabled (Grunewald, 2004). Unfortunately, data from intelligence tests did not exist for the population with ID in the present investigation. However, nothing indicated that the population was significantly different from the national average. Therefore, the WHO classification, paired with national ID prevalence figures, was used as the basis for estimation about likely test performance. About $80 \%$ of the population was estimated to be able interact reliably in ordinary pure-tone audiometry (OPTA) or 
conditioned play audiometry (CPA), methods generally managed from a mental age of five and three years, respectively (American Speech-Language-Hearing Association, 1997), while for the approximately $20 \%$ with severe/profound ID behavioural observation audiometry (BOA) should be available.

\begin{abstract}
Aim
The aim of this descriptive study was to evaluate in practice to what extent pure-tone screening audiometry with slight modifications could be applicable for testing a population with mild to profound intellectual disability.
\end{abstract}

\title{
MATERIAL
}

\section{Study group}

The project was carried out within a general hearing investigation of people with ID in a Swedish county. Approval of the project was given from the Research Ethics Committee, Linköping University (reg nr 90028) as well as from the County Secrecy and Integrity Committee (protocol 900301). The people with ID, their relatives and caring staff received, in written form, information about the project and invitation to the hearing investigation. If not accepting, a statement in writing was asked for. From the entire population of 1758 people in the age range $7-91$ years, registered to receive special support and service according to a mild, moderate, severe or profound intellectual disability, $1478(84.1 \%)$ people -850 males and 628 females - took part in the hearing screening carried out during 1992 - 1995 and 1998. 


\section{Excluded}

Pre-school children (age 0-6) with ID were excluded since those with DS were examined within directed habilitation programs while children with other ID aetiologies were in poor condition and their parents considered them unable to participate.

\section{Drop out from screening}

The reason why $280(15.9 \%)$ people out of the 1758 did not participate varied. Thirty-four earlier evaluated declined to participate. Two people were too ill to take part. One-hundredforty-four refrained from the screening since they or their significant others judged ears and hearing to be normal, they did not want to have any contact with the caring authority or, for non-specified reasons, they did not want to participate. Fifty-one moved from the county or died before the screening was completed, and another 49 registered in the county but living outside were not invited for practical reasons.

Age and gender of the screened population

Table 1 shows the age and gender distribution of the tested population.

\begin{tabular}{|l|c|c|c|c|c|c|c|c|c|c|c|}
\hline Age & $\mathbf{7 - 9}$ & $\mathbf{1 0 - 1 9}$ & $\mathbf{2 0 - 2 9}$ & $\mathbf{3 0 - 3 9}$ & $\mathbf{4 0 - 4 9}$ & $\mathbf{5 0 - 5 9}$ & $\mathbf{6 0 - 6 9}$ & $\mathbf{7 0 - 7 9}$ & $\mathbf{8 0 - 8 9}$ & $\mathbf{2 9 0}$ & Total \\
\hline Males & 33 & 205 & 143 & 159 & 139 & 92 & 49 & 25 & 5 & 0 & 850 \\
\hline & $2,2 \%$ & $13,9 \%$ & $9,7 \%$ & $10,8 \%$ & $9,4 \%$ & $6,2 \%$ & $3,3 \%$ & $1,7 \%$ & $0,3 \%$ & $0,0 \%$ & $57,5 \%$ \\
\hline Females & 17 & 128 & 110 & 111 & 109 & 87 & 42 & 19 & 4 & 1 & 628 \\
\hline & $1,2 \%$ & $8,7 \%$ & $7,4 \%$ & $7,5 \%$ & $7,4 \%$ & $5,9 \%$ & $2,8 \%$ & $1,3 \%$ & $0,3 \%$ & $0,1 \%$ & $42,5 \%$ \\
\hline Total & 50 & 333 & 253 & 270 & 248 & 179 & 91 & 44 & 9 & 9 & 1478 \\
\hline & $3,4 \%$ & $22,5 \%$ & $17,1 \%$ & $18,3 \%$ & $16,8 \%$ & $12,1 \%$ & $6,2 \%$ & $3,0 \%$ & $0,6 \%$ & $0,1 \%$ & $100,0 \%$ \\
\hline & & & & & & & & & & & \\
\hline & & & & & & & & & & & \\
\hline
\end{tabular}

Table 1. Age and gender distribution in the screened population $(n=1478)$ 
The larger prevalence in age groups between 10 and 49 years and the predominance of males is comparable to the distribution in the Swedish population with ID in total (Grunewald, 2004). Data concerning degree and type of ID had never been adequately assessed for the population. However, participants with DS were identified by ocular evidence and were estimated to constitute $13.7 \%$ of the screened population with age and gender distribution in parity with the screened population in total.

\section{Housing}

Private or official housing with special service as needed was the home for 758 participants (51.3\%), mostly adults, while $593(40.1 \%)$ participants, mostly children, lived with parents, other relatives or in foster homes. One-hundred-twenty-seven adults $(8.6 \%)$ had their own apartments.

\section{Activity}

In the screened population 403 participants (27.3\%) attended school, $895(60.5 \%)$ were engaged in day-activity centres, while $65(4.4 \%)$ worked in the open market and $32(2.2 \%)$ in sheltered workshops. Eighty-three participants (5.6\%) had no regular activity, of whom a majority were retired and/or seriously ill.

\section{METHOD}

\section{Instrumentation}

A pure-tone audiometer, Tegnér PTA 8, modified to present FM-tones was used. The standard TDH 39 earphones were complemented with insert earphones ER3A for use in narrow auditory canals. For participants who did not tolerate bilateral earphones, a single TDH 39 earphone, removed from the headset and held in the examiner's hand, was placed close to the 
subject's ear. The handheld procedure involved a risk of sound-leakage with influence on the test results. To estimate the risk and identify the correction values, if any, 48 ears in 12 normal hearing and 12 hearing-impaired people without ID were tested. Each ear was tested in a randomized order with headset as well as handheld earphone. There was a significant increase $(\mathrm{p}<0.001)$ of the hearing threshold level determined with the handheld procedure for 0.25 and $0.5 \mathrm{kHz}$, and a $-10 \mathrm{~dB}$ correction was needed.

The single earphone had a $5 \mathrm{~m}$ long flexible cable to allow following a participant moving around. For this purpose, a special remote control for the audiometer was also constructed which could be fastened with Velcro around the examiner's arm or leg.

For participants who rejected earphones, a small loudspeaker (diameter $60 \mathrm{~mm}$ ) was used to initially introduce the signals. If still rejecting earphones, screening was performed using the loudspeaker. An external power amplifier $(2.5 \mathrm{~W})$ was connected between the audiometer and the transducer and the equipment was calibrated using data presently included in ISO 389-7 (2005).

For conditioned play audiometry (CPA), an uncoloured wooden board with 25 blocks was used. The blocks were big enough to grip easily and many enough to admit testing of at least one ear without interruption. If easier for the participant, other manual response modes were accepted, e.g. putting balls into a bowl.

For visual reinforcement audiometry (VRA), a loudspeaker or bilateral earphones, a Panasonic Video Cassette Recorder NV-L25EO, a Panasonic Colour TV TC-1100EUN and pictures for clinical VRA testing were used. 


\section{Procedure}

The screening took place in schools, day-activity centres, sheltered workshops, and in some cases in group or private homes. Consequently, the test-rooms were quite different but fulfilled the requirements of being as silent as possible, having electricity, table, chairs, and space for a wheelchair. By subjective listening, the audiologist checked daily that the audiometer output was in order and that the room allowed screening at $20 \mathrm{~dB}$ (ISO 8253-1, 1989). Only one audiologist (author EA), experienced in working with ID people, performed the screening. As a basis for successful communication, the audiologist introduced herself during the participant's ordinary activity, and when contact was established the screening was performed in the test room. To make listening as free from interference as possible, caring staff or relatives were present only in exceptional cases.

FM (warble) tones with a modulation rate of $10 \mathrm{~Hz}$ and frequency deviation of $\sim 5 \%$ were used as test stimuli. The FM tones, easier to detect and less influenced by room acoustics than pure tones, were presented by earphones or from the loudspeaker placed at the distance $60 \mathrm{~cm}$ in front of the person (Arlinger \& Jerlvall, 1987; Magnusson et al., 1997; ISO 8253-2, 1992). The test mostly comprised the frequencies $0.25,0.5,1,2,4$ and $8 \mathrm{kHz}$ at the screening-level $20 \mathrm{~dB}$ HL. The test frequencies were limited to $0.5,1,2$ and $4 \mathrm{kHz}$ for less concentrated participants and for sound field screening. If the examiner judged the participant not to be able to co-operate even at these frequencies, 1, 2 and $4 \mathrm{kHz}$ on each ear were tested initially to secure screening data at least for the frequencies most important for speech recognition. When performing ordinary pure-tone audiometry (OPTA), a test tone at $40 \mathrm{~dB}$ or louder if needed was introduced initially to insure that the instruction was understood. Thereafter two test-tones at the screening level at each frequency were presented. If the participant failed, an additional tone was presented, and he/she passed if detecting two out of three. If the 
participant failed on screening at any frequency, the threshold was determined by increasing in $5 \mathrm{~dB}$ steps and decreasing in $10 \mathrm{~dB}$ steps until two valid responses were provided at the actual frequency (ISO 8253-1, 1989). The same test procedure was used for CPA, but as an introduction the examiner placed a wooden block in the participant's hand, laid her hand over it and showed how to put the block in the board when a stimulus was detected. As soon as the participant understood the procedure, the examiner took her hand away and the audiometry proceeded. When performing BOA by observation of body language responses, the stimulation was from start presented at the screening-level by bilateral earphones or loudspeaker.

In case of BOA by VRA, the auditory stimuli and reinforcing pictures were presented from the front via one loudspeaker and one TV-screen. This modification, not demanding lateral gaze orientation, aimed at making it easier for participants with motor difficulties to respond.

\section{Failure criterion}

The screening level was $20 \mathrm{~dB}$ HL and, if failing, threshold levels were determined.

Threshold levels $\geq 25 \mathrm{~dB}$ HL at two or more frequencies for one ear or both were ground for referral.

\section{RESULTS}

Of 1478 participants $1470(99.5 \%)$ co-operated in the screening. Eight $(0.5 \%)$ participants refused to fully co-operate, mostly out of fear, which was not even mitigated by the presence of a significant other. 


\section{Screened frequencies}

An absolute majority, 1325 (90\%) out of 1470 co-operating participants, could be tested at all six screening frequencies on both ears. In addition, 34 (2.3\%) could be tested on one ear, including those tested by loudspeaker. For $111(7.6 \%)$ participants with data at fewer frequencies, the missing frequencies were mostly 0.25 and $8 \mathrm{kHz}$. Based on the test results, average hearing threshold levels (HTL) for the four frequencies $0.5,1,2$ and $4 \mathrm{kHz}$ for the better ear were determined for $1462(99.5 \%)$ subjects.

\section{Transducers in screening}

A great majority, $1316(89.5 \%)$ of the co-operating participants, immediately accepted bilateral earphones or a single earphone. Additionally 79 (5.4\%), initially tested by loudspeaker, did accept one or two earphones as the screening session proceeded. Table 2 shows the types of transducers finally accepted.

\begin{tabular}{|c|c|c|c|c|}
\hline $\begin{array}{l}\text { Bilateral } \\
\text { earphones } \\
\text { TDH 39 }\end{array}$ & $\begin{array}{l}\text { Single } \\
\text { earphone } \\
\text { TDH 39 }\end{array}$ & $\begin{array}{l}\text { Insert } \\
\text { earphone }\end{array}$ & $\begin{array}{l}\text { Loudspeaker } \\
\text { ER3A }\end{array}$ & Total \\
\hline 1314 & 68 & 13 & 75 & 1470 \\
\hline $89,4 \%$ & $4,6 \%$ & $0,9 \%$ & $5,1 \%$ & $100,0 \%$ \\
\hline
\end{tabular}

Table 2. Types of transducers accepted in the screening $(n=1470)$

\section{Response methods in screening}

Of the co-operating participants, 987 (67.1\%) were tested by OPTA, 234 (15.9\%) by CPA, and 249 (16.9) by BOA.

In OPTA most participants responded by pressing a button or answering yes/now or humming "mm" but variations occurred - see Table 3 


\begin{tabular}{|c|c|c|c|c|c|c|c|}
\hline $\begin{array}{l}\text { Pressing } \\
\text { button }\end{array}$ & $\begin{array}{l}\text { Answering } \\
\text { yea/no/ } \\
\text { "mm" }\end{array}$ & $\begin{array}{l}\text { Raising } \\
\text { hand }\end{array}$ & $\begin{array}{l}\text { Pointing at } \\
\text { ear }\end{array}$ & $\begin{array}{l}\text { Imitating } \\
\text { stimulus }\end{array}$ & Nodding & $\begin{array}{l}\text { Closing } \\
\text { eyes }\end{array}$ & Total \\
\hline 483 & 438 & 40 & 11 & 10 & 4 & 1 & 987 \\
\hline $48,9 \%$ & $44,4 \%$ & $4,1 \%$ & $1,1 \%$ & $1,0 \%$ & $0,4 \%$ & $0,1 \%$ & $100,0 \%$ \\
\hline
\end{tabular}

Table 3. Ordinary pure-tone audiometry - response manner $(n=987)$

In CPA practically all participants responded by moving blocks. A few responded by moving things with which they were more familiar or could better handle, e.g. putting paper-clips in a box or dropping balls in a bowl.

For the participants screened with BOA, non-verbal, but sometimes vocal, communication was interpreted as responses. The response manners were to some extent over-lapping but could be divided into six specified groups (Table 4).

\begin{tabular}{|c|c|c|c|c|c|c|}
\hline $\begin{array}{l}\text { Eye } \\
\text { behaviour }\end{array}$ & $\begin{array}{l}\text { Minor } \\
\text { startle } \\
\text { reflex }\end{array}$ & $\begin{array}{l}\text { Vocal } \\
\text { behaviour }\end{array}$ & Posture & $\begin{array}{l}\text { Face } \\
\text { behaviour }\end{array}$ & Gesture & Total \\
\hline 80 & 68 & 55 & 26 & 16 & 4 & 249 \\
\hline $32,1 \%$ & $27,3 \%$ & $22,1 \%$ & $10,4 \%$ & $6,4 \%$ & $1,6 \%$ & $100,0 \%$ \\
\hline
\end{tabular}

Table 4. Behavioural observation audiometry - response manner $(n=249)$

BOA by VRA, designed to be used with severely or profoundly disabled participants, children as well as adults, turned out to be of marginal use because of difficulties in obtaining adequate and repeatable responses.

\section{Time consumption}

Time consumption was not assessed for each individual test session but approximately $97 \%$ of the participants were sufficiently introduced to the test within 2 to 5 minutes. More time consuming was to establish trust in anxious participants. The screening test typically required 
an additional 3 to 5 minutes but somewhat longer if threshold determination was needed for most frequencies, and for CPA if manipulation and vision difficulties occurred.

\section{Pass and fail in screening}

According to the referral criterion, 669 (45\%) participants passed and 809 (55\%) failed. The participants who failed were offered a clinical evaluation. However, only 441 of those failing took part in the evaluation. A majority among the 368 failing who did not accept referral or attend the evaluation did so because they as well as their significant others had not experienced any hearing problems.

Figure 1 illustrates the various stages of the study and the number of participants.

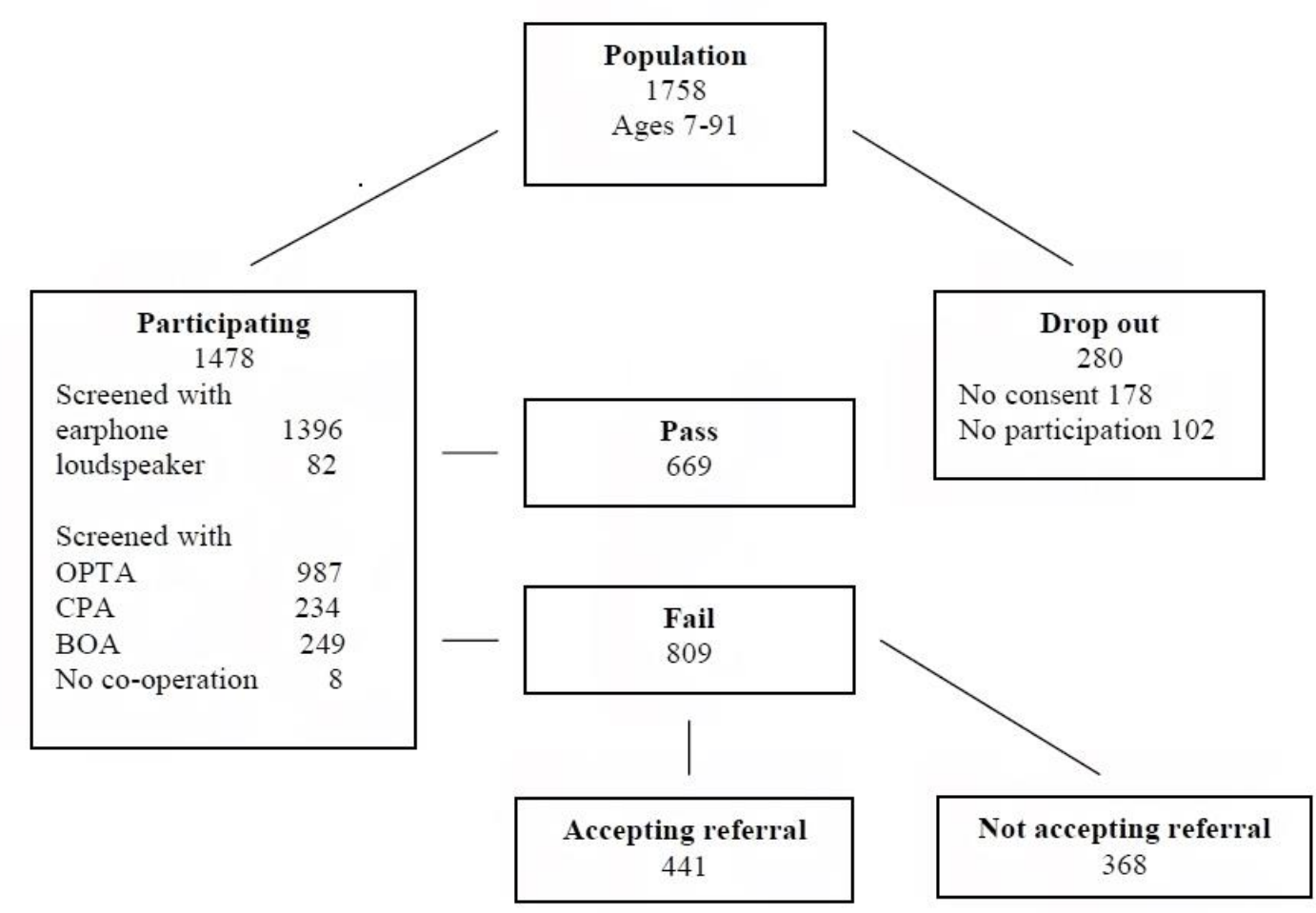

Figure 1. An overview of the number of participants in the screening procedure including number of pass and fail according to referral criteria. 


\section{DISCUSSION}

The need for assessment of auditory function of people with ID is well recognized (e.g.

Evenhuis \& Nagtzaam, 1998). However, there is no general agreement as to what test method is preferable. In the present study PTA was unambiguously shown to be useful in screening people with ID from school age to adults. According to criteria set by Wilson \& Jungner (1968) most of the general demands on an effective screening method were fulfilled. The participants acted as expected and about $80 \%$ of them acted in agreement with children and adults in general.

For the remaining 20\%, who were more difficult to test and mostly tested with BOA, the simple modifications and complements of the equipment made the test easier to perform. The small loudspeaker and the handheld single earphone were indispensible complements to the traditional equipment, not the least when introducing the screening procedure.

Recording of OAE might have been an alternative for the severely or profoundly disabled participants tested with BOA. OAE screening is proposed as the appropriate screening method for populations with ID (Gorga et al, 1995; Evenhuis \& Nagtzaam, 1998). However, Andersson et al (2000) showed in a study among 38 people with severe or profound ID, that the combination of the individual's inability to remain still and quiet and the equipment's sensitivity to noise negatively affected the result. Out of $89.5 \%$ failing, $55 \%$ showed a noisy recording or refused to participate and had to be re-tested or referred for evaluation together with the $34.5 \%$ with partial or no emissions. These facts prolonged the test procedure to on average $38 \mathrm{~min}$ per person while the OAE-recording itself took on average $3.2 \mathrm{~min}$. With comparable participants in the present study, the PTA procedure was in most cases successful and less time consuming. In addition, the comprehensive information from a PTA session - 
frequency specific hearing threshold data together with data about performance ability - may prevent referrals to unnecessary clinical evaluations.

BOA by VRA, despite being a common paediatric method, was not useful among the participants with severe or profound hearing loss for whom it was applied. It can be speculated why not. Vision and/or loco-motor problems may have affected the stimulus and/or response, the pictures presented might have been too complex or unknown to evoke an interest or too childish to stimulate an adult with ID. The modification with straight-ahead gaze orientation to stimulus instead of lateral orientation may have influenced the ability to interpret responses by the audiologist. Later, a better adapted reinforcement material was evaluated with nine adults with severe/profound ID to see if the modified method was more successful. A computer was used to present nonfigurative pictures, designed by an artist experienced in painting with ID people. However, only one participant of the nine tested gave adequate and reiterated eye behaviour responses that could be interpreted and reinforced.

The concern, anxiety and sometimes fear about what the test situation could involve encouraged some participants to seek security by sitting on the floor under a table or standing close to the door. Previous negative experiences might have influenced the test situation, and in order to succeed the audiologist must accept and even initiate an unconventional performance. It would have been too time consuming to insist on a formal "at-the-table" behaviour, and why insist when ordinary stimulus and response matters were functioning also under the circumstances mentioned. Soft kicks on the audiologist's shins, socks sorted into a tray, the comment "no, I won't", or a yawn for every signal heard were reactions as obvious as button pressing. The audiologist just needed an open mind, suitable clothes for floor sitting, 
long flexes and mobile furniture as well as a critical eye for what would constitute a repeatable response.

Not only were some participants sceptical of the screening situation, so were significant others. Despite generally very positive attitudes to screening and realizing the need for investigation, some declared: "X will not understand how to take part", or "X never cooperates with unknown people". Nevertheless, these apprehensions often came to naught since, as seen from the results, drop-outs due to lack of cooperation were very rare - in fact only eight people. Moreover, if the audiologist had shared an "it doesn't work" perspective and made decisions about limits beforehand, she would never have succeeded. The scepticism from the participants and significant others may be due to the rare occasion of hearing investigations making the procedure unfamiliar and sometimes frightening. These attitudes might change if a regular screening routine were used.

Before the actual study, no universal hearing screening had been carried out in the county, and now the goal was to detect and identify every person with any hearing loss. Consequently, a very strict pass/fail criterion was chosen and a large number of failing participants were detected. However, one third of them were not motivated to attend the clinical evaluation since they or their significant others did not notice any hearing problems. Unfortunately, with the non-attendance decision follows a risk for an unidentified hearing loss. Several studies have shown that judgements from significant others clearly underestimated the prevalence of hearing loss compared to results from audiometric screening, e.g. Lavis et al (1997), which partly can be explained by the fact that people with ID can not always communicate their hearing problems. In a population with ID in general, $70 \%$ are estimated to experience communication problems, receptive as well as expressive, and in a population with profound 
ID this figure amounts to $100 \%$ (Granlund, 1993). A screening protocol should contain information, emphasising that a hearing loss - even a minor - has negative effects on the communication for a person with ID, and everything possible should be done to facilitate optimal hearing. This should include also detecting a unilateral hearing loss.

The diversity of test methods and the infrequent use of PTA in hearing screening of people with ID is most likely due to the assumption that the population, from an audiological point of view, is "difficult to test", and particularly with PTA (Fulton \& Lloyd, 1975; Wilson \& Haire, 1990; Harris \& Dean, 2003).

Recording of OAE and ABR as well as tympanometry and surveys are, like PTA, common methods, but result in different audiological information. PTA provides information about a person's hearing, listening, communication and co-operating capability, forming a valuable basis for audiological (re)habilitation, while only limited conclusions about these characteristics can be drawn when using the other methods (Diefendorf, 2009; Schlauch \& Nelson, 2009).

The challenge is to find a course where the screening criteria with high sensitivity and specificity identify people with ID who need a referral for evaluation and treatment. The present study points out PTA as a candidate. A possible strategy for identification and habilitation might be a protocol based on PTA test at the frequencies $0.5,1,2,4 \mathrm{kHz}$ with the screening level $20 \mathrm{~dB}$ HL, and a referral limit of $25 \mathrm{~dB}$ HL or more at two or more frequencies for one ear or both. Offering people with ID a recurrent universal hearing screening program performed as proposed is possible to achieve in many countries. However, an obstacle deserving attention is divergent definitions of ID or limited possibilities to fully 
identify the population, which means divergent inclusion criteria and difficulties to compare results for epidemiological mapping.

Nevertheless, from an epidemiological point of view, a gold standard protocol for hearing screening in populations with ID is needed nationally and internationally. The protocol should be used in multi-centre studies aiming at estimating prevalence of hearing impairment as a basis for screening program, evaluation, re/habilitation and further research. Published results from hearing screening investigations can at present hardly be compared because of the disparity in screening methodology (e.g. Lavis et al, 1997; Driscoll et al, 2002; 2003; Meuwese-Jongejeugd et al, 2006; Neumann et al, 2006). Therefore, methodological advantages and/or disadvantages must be further analysed and discussed.

\section{CONCLUSIONS}

Access to best possible, universal, recurrent hearing screening can and must be offered people with ID to meet individual needs and to form a basis for further research concerning hearing in people with ID.

The present study shows that pure-tone screening audiometry with slight modifications is applicable for screening of a population with mild to profound intellectual disability. The most challenging and time-consuming activity is to introduce the test procedure in a way that reduces anxiety and establishes trust.

\section{ACKNOWLEDGEMENTS}

We gratefully acknowledge the financial support of the former Älvsborg County Council and the former Swedish Council for Social Research 


\section{DECLARATION OF INTEREST}

The authors report no conflict of interest. 


\section{REFERENCES}

American Speech-Language-Hearing Association 1997. Guidelines for Audiologic Screening. Retrieved: Dec 1, 2011 from http:॥ www.asha.org/policy.

Andersson E., Arlinger S. \& Jacobsson S. 2000. Evaluation of OAE-recording as a complementary test method for adults with moderate to profound mental retardation. Scand Audiol, 29, 120-126.

Arlinger S.D. \& Jerlvall L.B. 1987. Reliability in warble-tone sound field audiometry. Scand Audiol, 16, 21-27.

Davis A., Bamford J., Wilson I., Ramkalawan T., Forshaw M., et al. 1997. A critical review of the role of neonatal hearing screening in the detection of congenital hearing impairment. Health Technol. Assess, 1, i-iv, 1-176.

Diefendorf A.O. 2009. Assessment of hearing loss In: J. Katz, L. Medwetsky, R.F. Burkard \& L.J. Hood (eds.) Handbook of clinical audiology. Philadelphia: Wolters Kluwer Health/Lippincott Williams \& Wilkins, pp. 545-562.

Driscoll C., Kei J., Bates D. \& McPherson B. 2002. Transient evoked otoacoustic emissions in children studying in special schools. Int J Pediatr Otorhinolaryngol, 64, 51-60.

Driscoll C., Kei J., Bates D. \& McPherson B. 2003. Tympanometry and TEOAE testing of children with Down syndrome in special schools. Aust N Z J Aud, 25, 85-93. 
Evenhuis H. \& Nagtzaam L.M.D. 1998. IASSID International Consensus Statement: Early identification of hearing and visual impairment in children and adults with an intellectual disability. Leiden; Manchester: International Association of Scientific Studies on Intellectual Disability (IASSID) Special Interest Research Group on Health Issues; 1998. Retrieved: Dec 1, 2011 from www.iassid.org/pdf/consensir.alg.doc.

Evenhuis H.M., Theunissen M., Denkers I., Verschuure H. \& Kemme H. 2001. Prevalence of visual and hearing impairment in a Dutch institutionalized population with intellectual disability. J Intellect Disabil Res, 45, 457-464.

Fulton R.T. \& Lloyd L.L. 1975. Introduction. In: R.T. Fulton \& L.L. Lloyd (eds.) Auditory assessment of the difficult-to-test. Baltimore: Williams \& Wilkins, pp. xv, 297 p.

Gorga M.P., Stover L., Bergman B.M., Beauchaine K.L. \& Kaminski J.R. 1995. The application of otoacoustic emissions in the assessment of developmentally delayed patients. Scand Audiol Suppl, 41, 8-17.

Granlund M. 1993. Communicative competence in persons with profound mental retardation: intervention focused on the social context. Uppsala: Studia psychologica clinica Upsaliensia, ISSN 1100-3278; 3, Uppsala University.

Greenspan S. \& Granfield J.M. 1992. Reconsidering the construct of mental retardation: implications of a model of social competence. Am J Ment Retard, 96, 442-453.

Grunewald K. 2004. Utvecklingsstörningens medicinska orsaker. In: A. Bakk \& K. 
Grunewald (eds.) Omsorgsboken : en bok om människor med begåvningsmässiga funktionshinder. Stockholm: Liber, pp. 53-64

Harris F.P. \& Dean J. 2003. Introduction: Assessment of individuals with developmental disabilities. Semin Hear, 24, 169-170.

Hild U., Hey C., Baumann U., Montgomery J., Euler H.A., et al. 2008. High prevalence of hearing disorders at the Special Olympics indicate need to screen persons with intellectual disability. J Intellect Disabil Res, 52, 520-528.

Hodapp R.M., Burack J.A. \& Zigler E.F. 1998. Developmental approches to mental retardation: A short introduction. In: J.A. Burack, R.M. Hodapp \& E.F. Zigler (eds.) Handbook of mental retardation and development. Cambridge: Cambridge University Press, pp. 3-19.

ISO 389-7 2005. Acoustics - Reference zero for the calibration of audiometric equipment -Part 7: Reference threshold of hearing under free-field and diffuse-field listening conditions. Geneva: International Organization for Standardization.

ISO 8253-1 1989. Acoustics - Audiometric test methods -- Part 1: Basic pure tone air and bone conduction threshold audiometry. Geneva: International Organization for Standardization. 
ISO 8253-2 1992. Acoustics - Audiometric test methods - Part 2: Sound field audiometry with pure tone and narrow-band test signals. Geneva: International Organization for Standardization.

Kumar Sinha A., Montgomery J.K., Herer G.R. \& McPherson D.L. 2008. Hearing screening outcomes for persons with intellectual disability: a preliminary report of findings from the 2005 Special Olympics World Winter Games. Int J Audiol, 47, 399-403.

Lavis D., Cullen P. \& Roy A. 1997. Identification of hearing impairment in people with a learning disability: from questionnaire to testing. Brit J Learn Dis, 25, 100-105.

Magnusson L., Borjesson E. \& Axelsson A.C. 1997. Visual reinforcement audiometry. Comparison of loudspeaker arrangements. Scand Audiol, 26, 247-251.

Meuwese-Jongejeugd A., Vink M., van Zanten B., Verschuure H., Eichhorn E., et al. 2006. Prevalence of hearing loss in 1598 adults with an intellectual disability: cross-sectional population based study. Int J Audiol, 45, 660-669.

Neumann K, Dettmer G, Euler H.A., Giebel A., Gross M., et al. 2006. Auditory status of persons with intellectual disability at the German Special Olympic Games. Int J Audiol, 45, 83-90.

Rabinowitz P.M., Galusha D., Kirsche S.R., Cullen M.R., Slade M.D., et al. 2011. Effect of daily noise exposure monitoring on annual rates of hearing loss in industrial workers. Occup Environ Med, 68, 414-418. 
Schlauch R.S. \& Nelson P. 2009. Pure tone evaluation. In: J. Katz, L. Medwetsky, R.F. Burkard \& L.J. Hood (eds.) Handbook of clinical audiology. Philadelphia: Wolters Kluwer Health/Lippincott Williams \& Wilkins, pp. 30-49.

van Schrojenstein Lantman-de Valk H.M., Haveman M.J., Maaskant M.A., Kessels A.G., Urlings H.F., et al. 1994. The need for assessment of sensory functioning in ageing people with mental handicap. J Intellect Disabil Res, 38 ( Pt 3), 289-298.

White K.R., Vohr B.R., Maxon A.B., Behrens T.R., McPherson M.G., et al. 1994. Screening all newborns for hearing loss using transient evoked otoacoustic emissions. Int J Pediatr Otorhinolaryngol, 29, 203-217.

WHO 2010. ICD-10. Mental and Behavioural Disorders. Mental Retardation. Retrieved: Dec 1, 2011 from http://apps.who.int/classifications/apps/icd/icd10online/.

Wilson D.N. \& Haire A. 1990. Health care screening for people with mental handicap living in the community. BMJ, 301, 1379-1381.

Wilson J.M.G. \& Jungner Y.G. 1968. Principles and practice of mass screening for disease Public Health Papers No. 34. Geneva: WHO. Retrieved: Dec 1, 2011 from http://whqlibdoc.who.int/php/WHO_PHP_34.pdf. 